\title{
The relative distribution of dark matter and baryons in galaxy clusters
}

\author{
Andrea Biviano* \\ INAF/Osservatorio Astronomico di Trieste, Italy \\ E-mail: biviano@ts.astro.it
}

\section{Paolo Salucci}

SISSA, Trieste, Italy

E-mail: salucci@sissa.it

\begin{abstract}
Based on a data-set of about 3000 galaxies in 59 nearby clusters, and X-ray data for a subset of these clusters, we derive the average cluster baryonic mass profile, and the profile of the mass associated to cluster galaxies. We consider separately the contributions from cluster galaxies and the intra-cluster gas. We subtract the baryonic mass profile from the total mass profile, yielding the average cluster dark mass profile. We find that this profile is not very well fitted by the Navarro, Frenk, \& White profile, and a cored density profile provides a better fit. The baryon-todark mass profile is nearly constant, but shows an excess of baryons near the centre (due to the brightest cluster galaxies) and at large radii (due to the intra-cluster gas). A mass budget of the relative contribution of the different baryonic components and the dark matter is provided.
\end{abstract}

Baryons in Dark Matter Halos

5-9 October 2004

Novigrad, Croatia

${ }^{*}$ Speaker. 
The average mass profile of rich galaxy clusters has recently been determined by Katgert, Biviano, \& Mazure (2004, KBM hereafter), based on the ENACS data-set (Katgert et al. 1998). KBM found that the average cluster mass profile is well fit both by a profile of the form proposed by Navarro, Frenk, \& White (1997, NFW hereafter), characterized by an inner cusp, and by a profile of the form proposed by Burkert (1995, B95 hereafter), characterized by an inner core, as far as the size of the core-radius is rather small, below $\sim 140 \mathrm{kpc}^{1}$. However, the NFW profile, derived from cosmological numerical simulations with cold dark matter (DM) particles, describes the distribution of DM, while KBM determined the total mass profile of galaxy clusters. A proper comparison is between the NFW profile and the dark, not the total, mass profile of galaxy clusters. Here we determine the DM profile of galaxy clusters, based on the sample of KBM, and compare it to the NFW profile.

Following KBM, we combine 59 clusters into a single 'stacked' cluster, in order to improve upon the rather poor number statistics of individual clusters. For this, we scale individual galaxies clustercentric distances with the virial radii, $r_{200}$ 's, of the clusters which they belong to, and the individual galaxies line-of-sight velocities (with respect to the cluster mean) with the clusters line-ofsight velocity dispersions, $\sigma_{v}$. Using the results of numerical simulations, Sanchis, Łokas, \& Mamon (2004) have recently shown that stacking galaxy clusters indeed allows to reliably estimate the average mass profile of individual clusters. The stacked cluster extend to $\simeq 2.2 \mathrm{Mpc}=1.4\left\langle r_{200}\right\rangle$, the average value of $r_{200}$ for our 59 clusters being $1.6 \mathrm{Mpc}$.

With the procedure described in KBM, we determine the luminosity density profiles of earlytype and late-type galaxies. We then convert these profiles into baryonic mass density profiles, using the relations of van der Marel (1991), Persic, Salucci \& Stel (1996), and Fukugita, Hogan, \& Peebles (1998). The same luminosity density profiles are used to determine the subhaloes mass profile (i.e. the mass contained in galaxy haloes) by assuming the mass-to-luminosity relations displayed in Fig. 1.

In order to derive the intra-cluster (IC) gas baryonic mass profile, we use the sample of Xray clusters of Reiprich \& Böhringer (2002). From this sample we extract a subset of 59 clusters with the same X-ray temperature (i.e. $\sigma_{v}$ ) distribution as that of the 59 clusters in our sample (unfortunately, IC gas profiles are not directly available for all our 59 clusters). In Reiprich \& Böhringer (2002), the IC gas density profiles are parametrized by the usual $\beta$-model. We take the averages of the 59 best-fit $\beta$-model parameters $\left(\langle\beta\rangle=0.6\right.$ and $\left.\left\langle r_{X}\right\rangle=0.1 \mathrm{Mpc}\right)$, which then define the average cluster $\beta$-model. We integrate it to obtain an average IC gas mass profile, normalized in such a way as to provide 0.11 of the total mass at $r_{200}$ (Ettori, Tozzi, \& Rosati 2003). Note that extrapolation of the average gas profile is needed, since X-ray observations rarely cover the cluster regions out to the virial radius.

In Fig. 2 (top panel) we display the rotational velocity profiles, $V_{c}=\sqrt{G M / r}$, of the different cluster components. Two cluster DM profiles are shown: one is derived from the total mass by subtracting the baryonic mass only (black solid line in the figure), the other is derived by subtracting the baryonic mass and also the mass in subhaloes (black dash-dotted line in the figure). Approximate $1-\sigma$ confidence levels for the total mass profile are shown. The uncertainties on the total mass dominate the error budget on the DM profile. Clearly, baryons contribute very little to the total

\footnotetext{
${ }^{1}$ Throughout this paper we adopt $\mathrm{H}_{0}=70 \mathrm{~km} \mathrm{~s}^{-1} \mathrm{Mpc}^{-1}, \Omega_{m}=0.3$ and $\Omega_{\Lambda}=0.7$.
} 


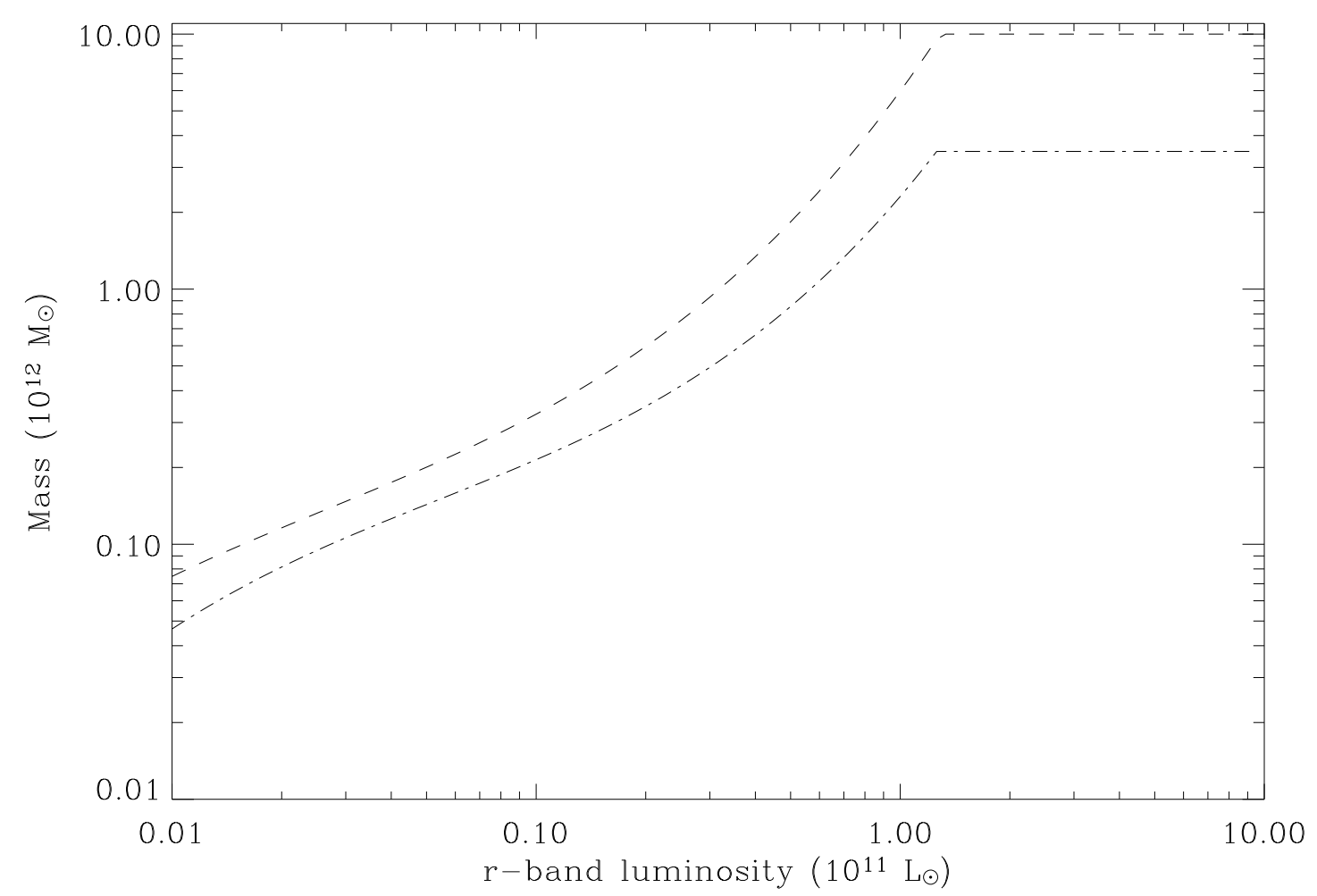

Figure 1: The conversion from galaxy luminosity to galaxy halo mass. Dashed line: early-type galaxies; dash-dotted line: late-type galaxies.

mass, and the DM profile is rather similar to the total mass profile (compare the solid black line with the solid blue line, respectively). However, a large component of the total mass could reside in individual subhaloes, which are especially relevant near the cluster centres.

In order to better illustrate the relative importance of the different cluster mass components, we show in Fig. 3 the ratios between the baryonic and the DM profiles. The total baryonic mass profile appears to be rather similar to the DM profile, their ratio being constant to within $\pm 20 \%$ within the virial radius. However, baryons in the IC gas are less centrally concentrated, and baryons in galaxies are more centrally concentrated, than the DM.

The fraction of DM in subhaloes is very high near the cluster centre, reaching up to $\sim 75 \%$, and then decreases outwards to an approximately constant value of $\sim 20 \%$. In principle it would seem unlikely that galaxies can retain their DM haloes close to the cluster centre, if anything because they would overlap. Tidal stripping is likely to be at work, as indicated by the results of numerical simulations (e.g. Gao et al. 2004). Nonetheless, most of the subhaloes mass near the centre is located in the haloes of $\mathrm{cD}$ galaxies. The very special place of $\mathrm{cD}$ galaxies near the bottom of the cluster potential well makes them less prone to tidal stripping. Hence, it is not unconceivable that indeed a large fraction of the cluster mass in the central regions is in subhaloes.

The cluster mass budget at the mean virial radius, $\left\langle r_{200}\right\rangle=1.6 \mathrm{Mpc}$ is the following: $2 \%$ of the mass is in galaxy baryons, $11 \%$ is in the IC gas baryons, and $87 \%$ is DM. Individual galaxy 


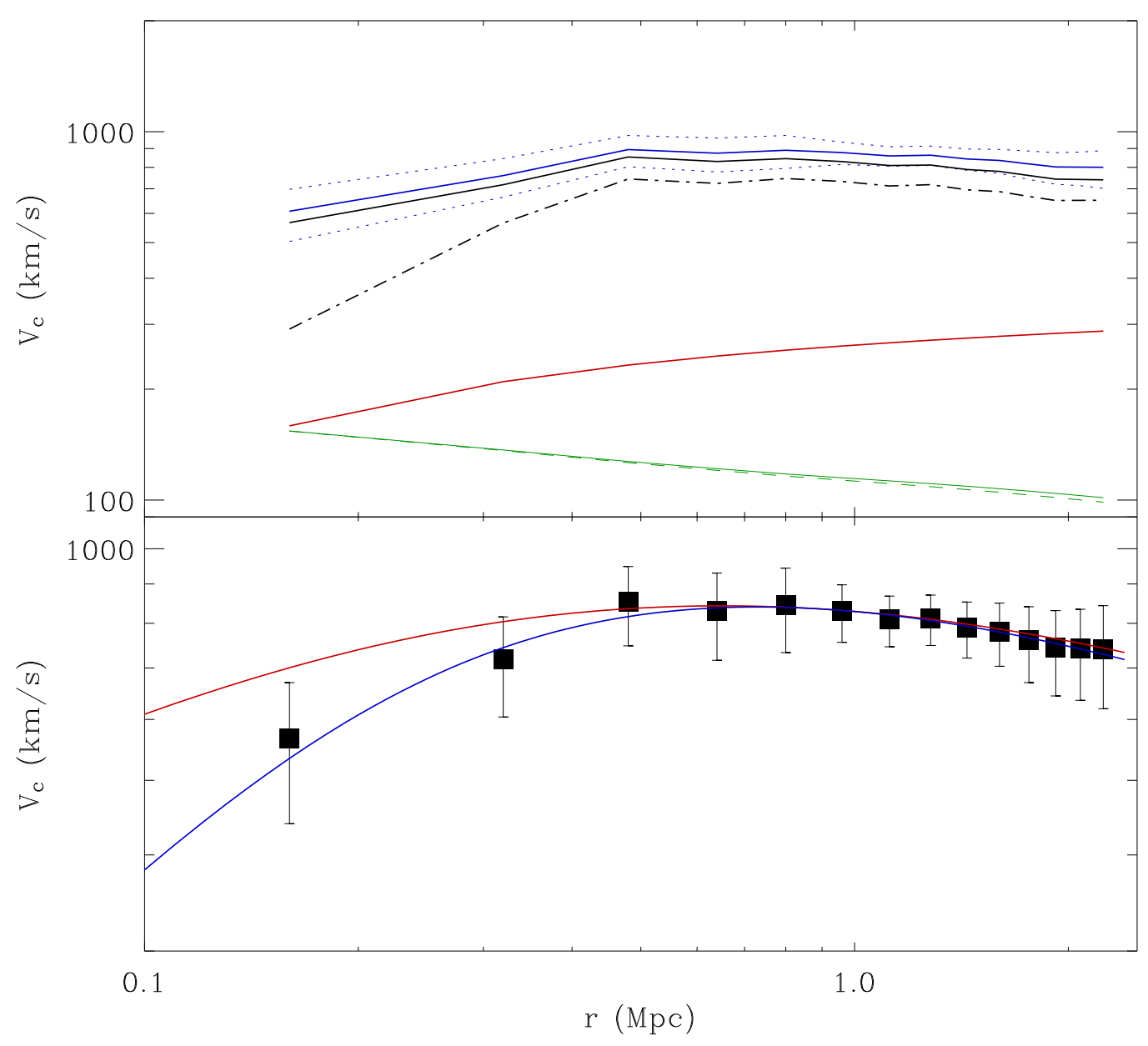

Figure 2: Top panel: the average cluster rotational velocity profiles. Blue lines: total mass (solid) and 1- $\sigma$ confidence levels (dotted). Black lines: DM (solid: total-baryons; dash-dotted: total-baryons-subhaloes). Red line: IC gas baryons. Green lines: galaxy baryons (solid: all galaxies; dashed: early-types only). Bottom panel: the average cluster rotational velocity profiles for the DM component only (black line), fitted by a NFW (red line), and a B95 (blue line) profiles.

haloes, if they survive the cluster environment, contribute $20 \%$ of the total mass. The baryon-tototal mass fraction in clusters, $\sim 13 \%$, is thus marginally below the universal value, $16.6 \pm 1.2 \%$ (Spergel et al. 2003), but it could approach the universal value at larger radii, where the IC gas mass fraction increases (see the dashed red line in Fig. 3).

The mass budget is drastically different near the cluster centre, at $0.1\left\langle r_{200}\right\rangle$, where the baryonic mass is almost equally shared between galaxies (6\%) and IC gas (7\%), although the DM still contributes the same $87 \%$ of the mass. If dark galaxy haloes were to survive near the centre they 


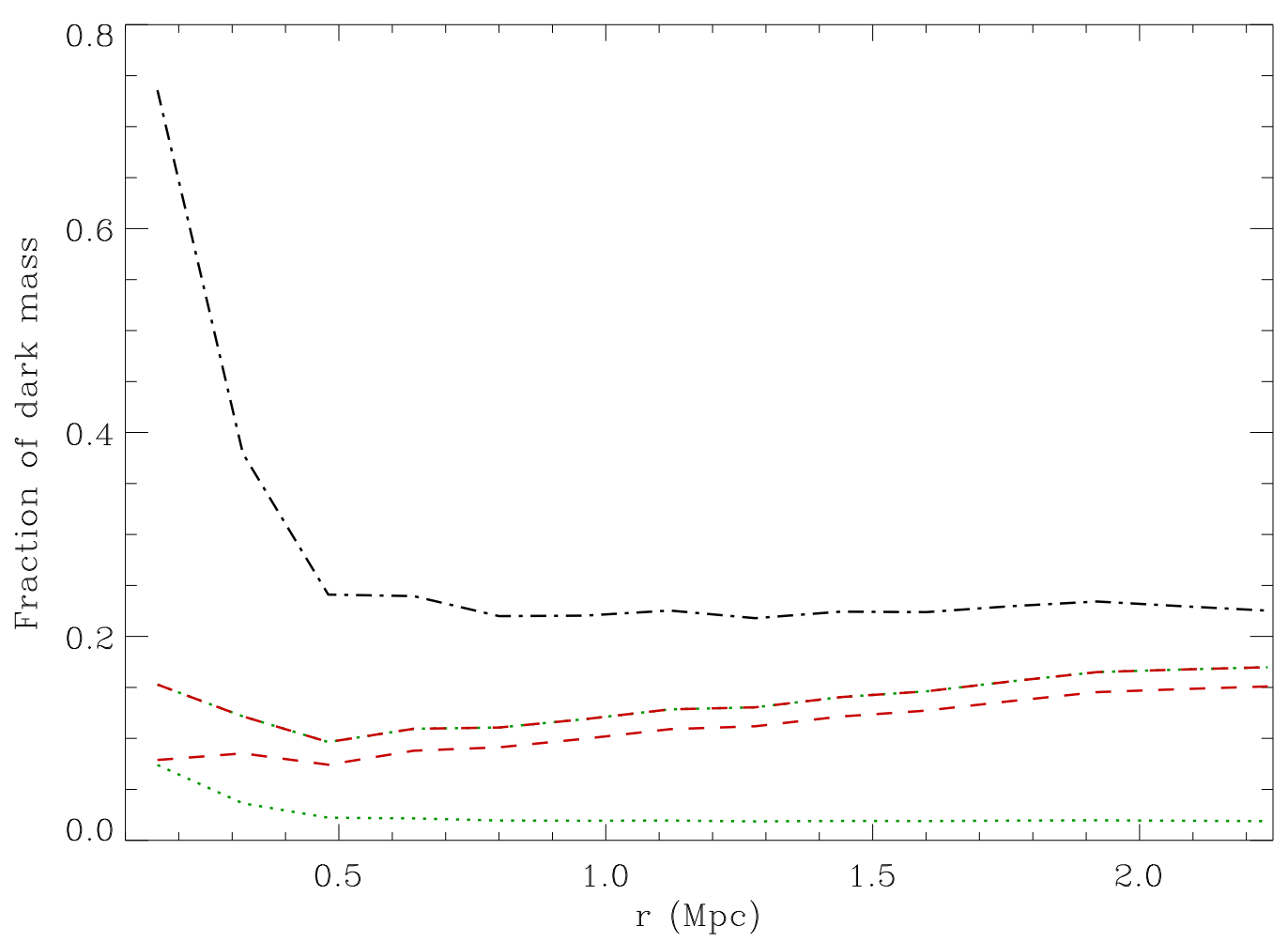

Figure 3: The ratio of the baryonic to the DM profiles. Green dotted line: (baryons in galaxies)/DM; red dashed line: (IC gas baryons)/DM; green and red dash-dotted line: (all baryons)/DM. The ratio of the subhaloes mass profile to the DM profile is shown as a black dash-dotted line.

would contribute most of the mass there, $64 \%$.

Subtracting the baryonic mass from the total mass makes the DM profile steeper than the total mass profile in the outer regions (where baryons in the IC gas are not a negligible mass component). As a consequence, the best-fit NFW profile (displayed as a red line in the bottom panel of Fig. 2) is more concentrated $(c=10)$ than the NFW profile fitting the total mass distribution $(c=4$, see $\mathrm{KBM}$ ). The concentration must increase to fit the outer profile, but then the NFW profile becomes too steep near the centre. A B95 profile provides a better fit to the DM distribution (see the blue line in the bottom panel of Fig. 2) The best-fit core radius of the B95 profile is $0.225 \mathrm{Mpc}$, significantly larger than the value found by KBM for the total mass distribution $(0.15 \mathrm{Mpc})$.

We conclude that the NFW profile has some difficulties to represent the cluster DM profile, which seems to be characterized by a central core.

\section{References}

[1] Arnaud, M., \& Evrard, A.E. 1999, MNRAS, 305, 631

[2] Burkert, A. 1995, ApJ, 447, L25 (B95)

[3] Ettori, S., Tozzi, P., \& Rosati, P. 2003, A\&A, 398, 879 
[4] Fukugita, M., Hogan, C.J., \& Peebles, P.J.E. 1998, ApJ, 503, 518

[5] Gao, L., De Lucia, G., White, S.D.M., \& Jenkins, A. 2004, MNRAS, 352, L1

[6] Katgert, P., Biviano, A., \& Mazure, A. 2004, ApJ, 600, 657 (KBM)

[7] Katgert, P., Mazure, A., den Hartog, et al. 1998, AAS, 129, 399

[8] Navarro, J.F., Frenk, C.S., \& White, S.D.M. 1997, ApJ, 490, 493 (NFW)

[9] Persic, M., Salucci, P., \& Stel, F. 1996, MNRAS, 281, 27

[10] Reiprich, T.H. \& Böhringer, H. 2002, ApJ, 567, 716

[11] Sanchis, T., Łokas, E.L., \& Mamon, G.A. 2004, MNRAS, 347, 1198

[12] Spergel, D.N., Verde, L., Peiris, H.V., et al. 2003, ApJS, 148, 175

[13] van der Marel, R., 1991, MNRAS, 253, 710 